\title{
Diagnostic performances of manual and automated reticulocyte parameters in anemic cats
}

\begin{tabular}{|c|c|}
\hline Journal: & Journal of Feline Medicine and Surgery \\
\hline Manuscript ID & JFMS-16-0144.R2 \\
\hline Manuscript Type: & Original Article \\
\hline Date Submitted by the Author: & $n / a$ \\
\hline Complete List of Authors: & $\begin{array}{l}\text { Paltrinieri, Saverio; University of Milan, Veterinary Medicine } \\
\text { Fossati, Marco; Veterinary Clinic Artemedica } \\
\text { Menaballi, Valentina; University of Milan, Veterinary Medicine; Veterinary } \\
\text { Clinic Artemedica }\end{array}$ \\
\hline Keywords: & $\begin{array}{l}\text { anemia, diagnostic accuracy, feline, reticulocyte number, reticulocyte } \\
\text { percentage, reticulocyte production index }\end{array}$ \\
\hline Abstract: & 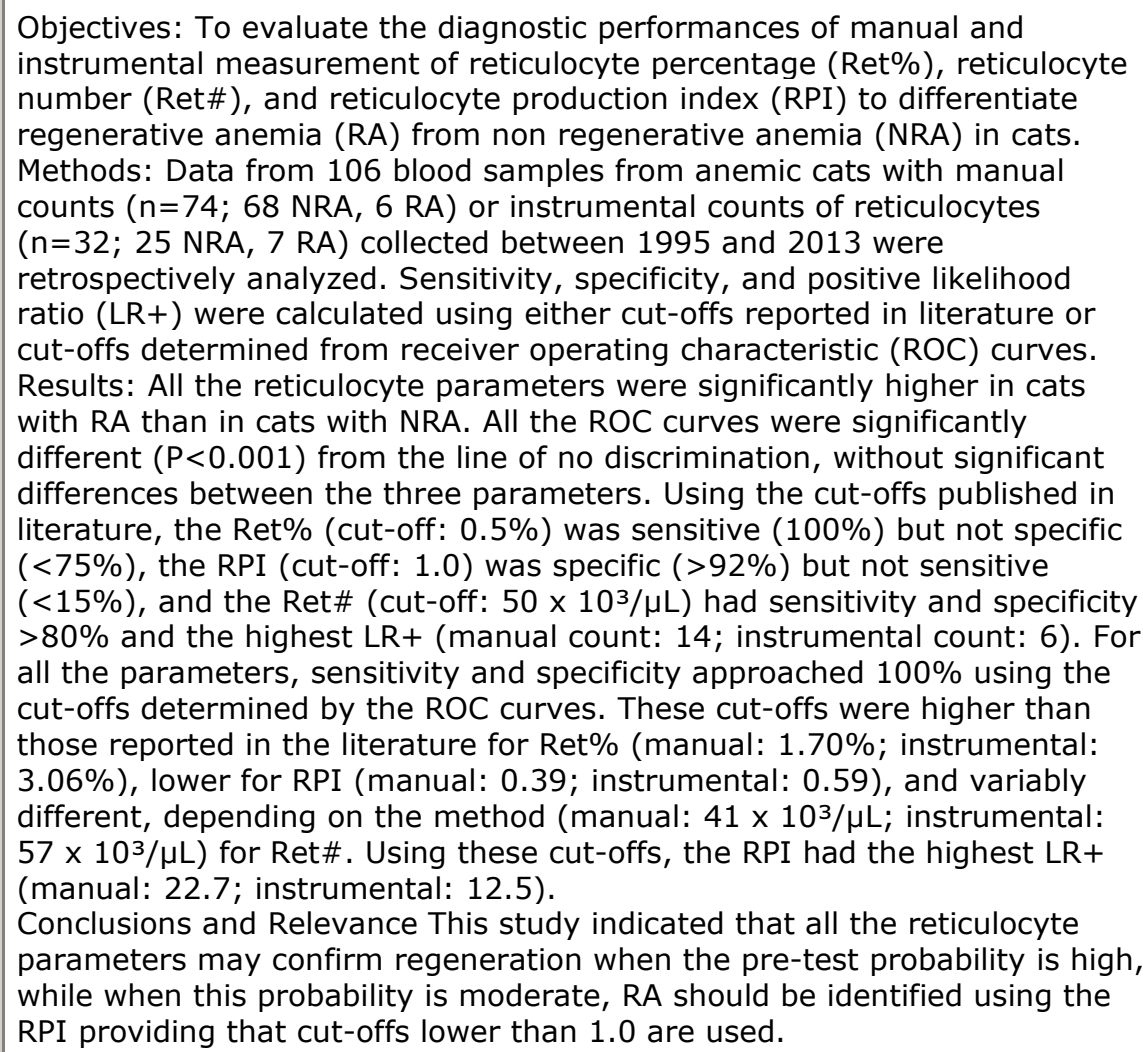 \\
\hline
\end{tabular}


SCHOLARONE $^{\text {m }}$
Manuscripts 
1 Diagnostic performances of manual and automated reticulocyte parameters in anemic

2 cats

3

4 Paltrinieri S., Dept. of Veterinary Medicine, University of Milan, 20133, Milan, Italy

$5 \quad$ Fossati M., Veterinary Clinic Artemedica, Valmadrera, 23868, Italy

6 Menaballi V., Dept. of Veterinary Medicine, University of Milan, 20133, Milan, Italy, and

7 Veterinary Clinic Artemedica, Valmadrera, 23868, Italy

8

9

10 Corresponding Author:

11 Prof. Saverio Paltrinieri, DVM, PhD, Dipl ECVCP

12 Dipartimentto di Medicina Veterinaria,

13 Università degli Studi di Milano

14 Via Celoria 10, 10133, Milano

15 Tel. 0250318103,

16 Fax: 0250318095 
17 saverio.paltrinieri@,unimi.it

18

19 Keywords:

20 Anemia; Diagnostic accuracy; Feline; Reticulocyte number; Reticulocyte percentage;

21 Reticulocyte production index;

22 


\section{Abstract}

Objectives: To evaluate the diagnostic performances of manual and instrumental measurement of reticulocyte percentage (Ret\%), reticulocyte number (Ret\#), and reticulocyte production index $(\mathrm{RPI})$ to differentiate regenerative anemia (RA) from non regenerative anemia (NRA) in cats.

Methods: Data from 106 blood samples from anemic cats with manual counts $(n=74 ; 68$ NRA, 6 RA) or instrumental counts of reticulocytes ( $n=32 ; 25$ NRA, 7 RA) collected between 1995 and 2013 were retrospectively analyzed. Sensitivity, specificity, and positive likelihood ratio $(\mathrm{LR}+)$ were calculated using either cut-offs reported in literature or cut-offs determined from receiver operating characteristic (ROC) curves.

Results: All the reticulocyte parameters were significantly higher in cats with RA than in cats with NRA. All the ROC curves were significantly different $(\mathrm{P}<0.001)$ from the line of no discrimination, without significant differences between the three parameters. Using the cut-offs published in literature, the Ret\% (cut-off: 0.5\%) was sensitive (100\%) but not specific ( $<75 \%$ ), the RPI (cut-off: 1.0$)$ was specific $(>92 \%)$ but not sensitive $(<15 \%)$, and the Ret\# (cut-off: $50 \times 10^{3} / \mu \mathrm{L}$ ) had sensitivity and specificity $>80 \%$ and the highest LR+ (manual count: 14; instrumental count: 6). For all the parameters, sensitivity and specificity approached $100 \%$ using the cut-offs determined by the ROC curves. These cut-offs were 
41 higher than those reported in the literature for Ret $\%$ (manual: $1.70 \%$; instrumental: $3.06 \%$ ),

42 lower for RPI (manual: 0.39; instrumental: 0.59), and variably different, depending on the Comment [a2]: Removed: dependently

43 method (manual: $41 \times 10^{3} / \mu \mathrm{L}$; instrumental: $57 \times 10^{3} / \mu \mathrm{L}$ ) for Ret\#. Using these cut-offs, the

44 RPI had the highest LR+ (manual: 22.7; instrumental: 12.5).

45 Conclusions and Relevance This study indicated that all the reticulocyte parameters may

46 confirm regeneration when the pre-test probability is high, while when this probability is

47 moderate, RA should be identified using the RPI providing that cut-offs lower than 1.0 are 48 used.

Comment [a3]: Removed: at 


\section{Introduction}

The differentiation between regenerative anemia (RA) and non regenerative anemia (NRA) may drive further diagnostic or therapeutic procedures. ${ }^{1}$ The identification of RA relies on the quantification of reticulocyte responses. To this aim, the current literature recommends to use the absolute number of reticulocytes (Ret\#) rather than the reticulocyte percentage (Ret\%) or the reticulocyte production index (RPI)., ${ }^{2,3}$ However, the cut-offs reported in the literature for feline Ret\# are variable (e.g. $40-60 \times 10^{3} / \mu \mathrm{L}$ ) and have been determined using different methods, including laser-based counters, that have a higher analytical sensitivity and provide higher Ret\# compared with manual counts. ${ }^{2,4-8}$ Moreover, the Ret\# and the Ret $\%$ are higher in some feline breeds than in others (the Ret $\%$ may be as high as $0.8 \%$ in Norwegian Forest cats, $1.2 \%$ in Holy Birman cats, $1.9 \%$ in Siberian cats and 3.3\% in Maine Coon cats, and the Ret\# may be as high as 250.00 in Main Coon cats) $)^{9,10}$ Additionally, the

magnitude of reticulocytosis should inversely correlate with the severity of anemia. ${ }^{2,11}$ In

human medicine the RPI has been proposed as a tool to correct the Ret $\%$ for the severity of anemia. ${ }^{3}$ The calculation of RPI is based on the maturation time of human circulating reticulocytes, that is higher in RA, when reticulocytes released in blood are younger than in healthy individuals. ${ }^{3}$ The maturation time of feline reticulocyte is unknown but it is likely different from other species, since feline erythroid cells have some peculiarities such as a 
shorter erythrocyte lifespan, a prolonged maturation time of punctate reticulocytes and a weaker maximal response of aggregate reticulocytes. ${ }^{12}$ Independent of the correctness of the formula we recently demonstrated that the Ret $\%$ and the RPI may be used to diagnose canine RA. ${ }^{13}$ However, no studies on the utility of the three reticulocyte parameters in cats are available.

Hence, this study was aimed to assess the diagnostic performances of Ret $\%$, Ret\# and RPI counted manually or by a laser-based analyzer, for the diagnosis of RA in cats.

\section{Materials and methods}

\section{Case selection criteria}

The laboratory information system was analyzed to retrieve data recorded between January 1995 and January 2013 from anemic cats. The database included data generated using an impedance counter (SEAC Hemat 8) followed by reticulocyte counts on brilliant cresyl blue stained smears, or using a laser counter validated in cats (Sysmex XT2000-iV). ${ }^{14,15}$ Only aggregate reticulocytes were counted manually, since punctate reticulocytes do not indicate active or recent regeneration in cats. ${ }^{12}$ Moreover, a moderate to high correlation between aggregate reticulocyte counts and automated reticulocyte counts|with Sysmex |has previously been reported. ${ }^{14,15} \|$ 
The inclusion criteria were the following: presence of anemia based on the comparison with the reference intervals in use at our institution for cats $\left(\mathrm{RBC}<5.0 \times 10^{3} / \mu \mathrm{L},|\mathrm{Ht}<27 \%,| \mathrm{Hb}\right.$ $<10 \mid \mathrm{g} / \mathrm{dL})$; availability of manual or instrumental reticulocyte counts; availability of stored glass slides to review the original classification; |diagnosis of RA or NRA based on history, diagnostic tests (necropsy, cytology; serum biochemistry, bone marrow cytology; serology/PCR for infectious diseases) or follow up (recovery within one month for RA; no improvement during one year follow up for NRA).| Post-hemorrhagic acute anemia was classified in the RA group when sampling were done at least 5 days after the hemorrhage, or within the NRA group when sampling were done 1-2 days after the hemorrhage|(preregenerative phase of acute anemia).

Samples from cats treated with drugs that influence bone marrow activity, or belonging to breeds known to have high reticulocyte counts ${ }^{9,10}$ were excluded from the study.
Comment [a15]: Removed: Institution Comment [a16]: Removed: ;

Comment [a17]: Removed: 37 Comment [a18]: Removed: ; Comment [a19]: Removed: 12 Comment [sp20]: Removed: in doubt cases

Comment [a21]: Removed: Independent of the time of recovery,

Comment [a22]: Removed: hemorrhages occurred at least 5 days before sampling

Comment [a23]: Removed: hemorrhages occurred 1-2 days before sampling

\section{Calculation of reticulocyte parameters}

Based on RBC numbers and on the Ret\% generated by manual or instrumental counts, the following parameters were calculated using the formula reported in literature: ${ }^{3}$

- $\quad$ Ret $\#=$ number of erythrocytes $x(\operatorname{Ret} \% / 100)$

- Corrected $\operatorname{Ret} \%=\operatorname{Ret} \% \mathrm{x}(\mathrm{Hct} / 37)$

Comment [a25]: Removed: 45 
- $\quad$ RPI $=$ corrected $\operatorname{Ret} \% /[(-0.05) \times$ Hct +3.25$]$

105 Fact that the maturation time of feline reticulocytes is unknown, the RPI was calculated

106 using the formula used in people, ${ }^{3}$ assuming that also in cats the maturation time is longer if

\section{Statistical analysis}

112 Data from manual and instrumental counts were analyzed separately. The Ret\%, Ret\# and RPI

113 for each group (RA, NRA) were compared with the Friedman test with the Bonferroni

114 correction using a commercial software (Analyse-it Ltd).

115 For each parameter, the number of true positives and false negative (samples with RA with

116 values higher and lower, respectively, than each operating point), true negative and false

117 positive (samples with NRA with values lower and higher, respectively, than each

118 operating point), were calculated. ${ }^{16}$ Sensitivity, specificity and positive likelihood ratio

$119(\mathrm{LR}+)$ were calculated using standard formulae ${ }^{17}$ either at the cut-offs determined with the

120 receiver operating characteristic (ROC) curves, or using the upper reference limits reported

121 in the literature $\left(\operatorname{Ret} \%=0.5 \%\right.$; Ret $\left.\#=50 \times 10^{3} / \mu \mathrm{L} ; \mathrm{RPI}=1.0\right){ }^{2} \mid \mathrm{ROC}$ curves were designed 
122 to determine the ability of each parameter to identify cats with RA and to identify which

123 cut-off best differentiates RA from NRA. ${ }^{18}$ The level of significance in all the statistics

124 above was set at $\mathrm{P}<0.05$

125

126 Results

127 Composition of groups

128 The retrospective search in the database according to the selection criteria (figure 1)

129 allowed us to include $\mid 106$ cases in the study (table 1) $\mid$ The RA group (table 1) $\mid$ included cats

130 with hemoplasma infection or|with acute hemorrhage occurred at least 5 days before

131 sampling. The NRA group included either recent hemorrhage or infectious, metabolic or

132 neoplastic conditions that depress the bone marrow activity.

135 With both methods, results for all the reticulocyte parameters were significantly higher in

136 cats with RA than in cats with NRA (figure 2). In cats with RA, the Ret\%, Ret\# and RPI

137 were higher than the upper reference limit reported in literature ${ }^{2}$ in $6 / 6,4 / 6$ and $0 / 6$ cases

138 after manual counts and in 6/6,0/7 and 6/7 cases after instrumental count. In the NRA 
139 group, the Ret $\%$, Ret\# and RPI were abnormal in 13/68, 4/68 and 0/68 cats after manual

140 counts and in $7 / 25,4 / 25$ cats and $2 / 68$ cats after instrumental counts.

141 Hence, using both methods, the Ret\% was more sensitive than specific, the RPI was very

142 specific but not sensitive, and the Ret\# had sensitivity and specificity $>80.0 \%$ and the

143 highest LR+ (table 2). However, for all the parameters, sensitivity and specificity

144 approached $100.0 \%$ if cut-offs determined by the ROC curves were used. These cut-offs

145 were higher than those reported in the literature for Ret $\%$, lower for RPI, and variably

146 different, depending on the method used to enumerate the reticulocytes, for Ret\#. Using

Comment [sp41]: Removed: dependently

147 these cut-offs, the RPI had the highest LR+ using both methods. However, the

148 discriminating power of all the parameters, as defined by the ROC curves (figure 3) was

149 always close to $100 \%$ and significantly higher $(\mathrm{P}<0.001)$ than the line of no discrimination,

Comment [a42]: Removed: .0

150 without significant differences between the three parameters.

\section{Discussion}

153 This study indicated that all the reticulocyte parameters may identify cats with regenerative

154 anemia. Moreover, the different counting methods provided similar results, contrary to

155 what occurred in dogs. ${ }^{13} \mid$ This was likely due to the fact that |instrumental counts mostly

156 detect aggregate reticulocytes ${ }^{15}$ (i.e the only cells that are counted manually in cats) and not

Comment [a43]: Removed: regeneration
Comment [a44]: Removed: differently from
Comment [sp45]: Removed: contrarily to
Comment [a46]: Removed: 12
Comment [a47]: Removed: because


157 punctate reticulocytes that are included in manual counts in dogs. However, all the

158 reticulocyte parameters had better performances if cut-offs different from those reported in

159 the literature are used. This is particularly true for the RPI, that corrects the magnitude of

160 reticulocytosis for the severity of anemia. ${ }^{2,3}$ The RPI is based on the maturation time of

161 human reticulocytes, which is likely different than in cats, due to the peculiarities of feline

162 erythroid cells. ${ }^{12}$ However, it is very likely that also in cats the maturation times of

163 circulating reticulocytes increases if these cells are released earlier than in normal

164 conditions. Hence, we assessed whether RPI, as well as the other reticulocyte parameters,

165 may provide useful diagnostic information at the cut-off reported in the literature or at a

166 different cut-off, identified through a ROC curve analysis. This approach revealed that |the

167 cut-off reported in the literature for humans is very specific but not sensitive. This is not

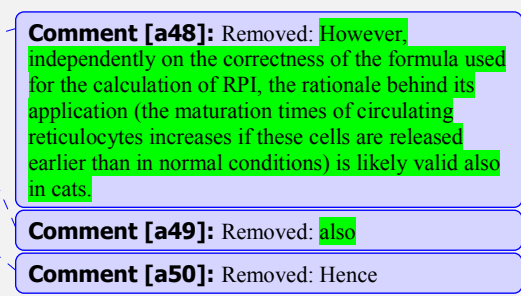

168 surprisingly, based on the peculiarities of feline reticulocytes described in the

169 introduction. ${ }^{12}$ Hence, further studies on the maturation time of feline reticulocytes are

170 needed in order to increase the accuracy of the formula used to calculate the RPI in cats.|

171 However, using lower cut-offs, the RPI had the highest LR+. A similar finding was

Comment [a51]: Removed: and confirms that the formula for calculating the feline RPI should be

172 recorded in dogs, ${ }^{13}$ which also have different maturation times compared with humans.

173 Although the ROC curves did not detect significant differences between the discriminating

174 power of the three parameters, the RPI, at cut-offs lower than those of humans, is preferred 
175 if the pre-test probability of regeneration is unknown, since in this case a test with high

176 LR+ increases the post-test probability of disease. ${ }^{16,18}$ Conversely, if the pre-test probability

177 of regeneration is high (evident blood loss or hemolysis) a test with high specificity, that

178 avoids false positive results, may be appropriate las a confirmatory test. Based on our

179 results, any reticulocyte parameter, except the Ret $\%$ at the cut-off reported in literature,

180 may play this confirmatory role.

181 The main limitation of this study is the low number of cats, especially in the RA group.

182 However, the performance of each parameter was expressed in terms of $\mathrm{LR}+$, that,

183 differently from predictive values, is not affected the prevalence of the diseases, ${ }^{18}$ thus

184 minimizing the effect of the low number of cats with RA. The prevalence of RA in our caseload ranged from $8 \%$ (manual counts) to approximately $20 \%$ (instrumental counts). although recent reports suggest that hematologic patterns consistent with RA or pathogenic mechanisms responsible for RA are present in more than $40 \%$ of anemic cats. ${ }^{8,20}$ However, the latter studies were based on cut-offs that, according to the current study are poorly specific and may have overestimated RA. The low prevalence of RA in our study, and the

191 lack of cases of immune-mediated hemolytic anemia, were likely due to the application of 
193 multiple pathogenic mechanisms or by inaccurate classification of RA. This is important

194 since the bone marrow cytology, that is considered the most accurate marker to assess the

195 presence of regeneration||is not recommended in routine practice when blood loss or hemolysis are suspected based on clinical or hematological findings..$^{2}$ Hence, cats were

classified based on the final diagnosis and on the restoration of the RBC mass during the

follow up, as in a similar study in dogs. ${ }^{13} \mid$ To this aim, we established a long time limit to assess whether restoration of the RBC mass occurred or not (one year), to not misclassify cases of regenerative anemia in the NRA group. Independent of the time of recovery, acute post-hemorragic anemia was included in the NRA group when samples were obtained in the first days after the hemorrhage, since during pre-regenerative anemia reticulocytosis is not fully detectable in peripheral blood. Conversely, in the similar study in dogs, ${ }^{13}$ the ability of reticulocyte parameters to detect regeneration was assessed either including preregenerative anemia in the NRA group, to detect full regeneration, or in the RA group, to detect early regeneration. However, in the current study this approach was hampered by the low number of cases with pre-regenerative anemia. Future studies including higher number of cases and especially a higher number of cats with of pre-regenerative anemia are needed to confirm that Ret\#, Ret\% or RPI may be early indicators of regeneration in cats.
Comment [a58]: Removed: (the presence of erythroid hyperplasia in bone marrow)

Comment [a59]: Removed: is diagnosed

Comment [a60]: Removed: 12
Comment [a61]: Removed: The only exception to this rule regarded post-hemorrhagic anemia, that was included in the NRA group when sampling were performed in the first days after the hemorrhagic event
Comment [a62]: Removed: It would be interesting, in the future, to extend this study to a higher number of cases of pre-regenerative anemia in order to assess if Ret\#, Ret\% or RPI may be early indicators of regeneration as it was the case in dogs. 
210 In conclusion, this study indicated that all the reticulocyte parameters confirm regeneration

211 when the pre-test probability is high, while when this probability is moderate, RA should

212 be identified using the RPI at cut-offs |lower than 1.0.|

Comment [a63]: Removed: notably Comment [a64]: Removed: However, further studies on a higher caseload should be encouraged to support the results of the current study.

\section{Acknowledgment}

215 Part of this study was presented at the $15^{\text {th }}$ Conference of the European Society of

216 Veterinary Clinical Pathology and the European College of Veterinary Clinical Pathology,

217 Berlin, Germany, October 2013.

218

\section{Funding}

220 The authors received no specific grant from any funding agency in the public, commercial 221 or not-for-profit sectors for the preparation of this article.

\section{Conflict of interest}

224 The authors do not have any potential conflicts of interest to declare 


\section{References}

1. Cowgill ES, Neel JA and Grindem CB. Clinical application of reticulocyte counts in dogs and cats. Vet Clin North Am Small Anim Pract 2003; 33: 1223-1244.

2. Tvedten H. Laboratory and clinical diagnosis of anemia. In: Weiss DJ and Wardrop KJ (eds). Schalm's Veterinary Hematology. $6^{\text {th }}$ ed. Ames, IA: Wiley-Blackwell, 2010, pp. 152-161.

3. Christian JA. Erythrokinetics and erythrocyte destruction. In: Weiss DJ and Wardrop KJ (eds). Schalm's Veterinary Hematology. $6^{\text {th }}$ ed. Ames, IA: Wiley-Blackwell, 2010, pp. 136-143.

4. Weiss DJ and Tvedten H. Erythrocyte disorders. In: Willard MD and Tvedten H (eds). Small animal clinical diagnosis by laboratory methods. $5^{\text {th }}$ ed. St Louis, MO: Elsevier Saunders, 2012, pp. 38-62.

5. Weingart C, Tasker S, and Kohn B. Infection with haemoplasma species in 22 cats with anaemia. J Feline Med Surg. 2016; 18: 129-136

6. Moritz A, Fickenscher Y, Meyer K, et al. Canine and feline hematology reference values for the ADVIA 120 hematology system. Vet Clin Pathol 2004; 33: 32-38.

7. Weissenbacher S, Riond B, Hofmann-Lehmann R et al. Evaluation of a novel haematology analyser for use with feline blood. Vet J. 2011; 187: 381-387. 

Samples with Anemia from Adult Cats: Frequency, Classification, and Association with Serum Creatinine Concentration. J Vet Intern Med 2014; 28: 1391-1397.

9. Spada E, Antognoni MT, Proverbio D, et a1. Haematological and biochemical reference intervals in adult Maine Coon cat blood donors. J Feline Med Surg. 2015; 17: $1020-1027$.

10. Paltrinieri S, Ibba F and Rossi G. Haematological and biochemical reference intervals of four feline breeds. J Feline Med Surg. 2014;16 :125-136.

11. Riley RS, Ben-Ezra JM, Goel R, et al. Reticulocytes and reticulocyte enumeration. $J$ Clin Lab Anal 2001; 15: 267-294.

12. Cramer DV and Lewis R . Reticulocyte response in the cat. J Am Vet Med Assoc $1972 ; 160: 61-66$.

13. Paltrinieri S, Rossi G., Manca M et al. Sensitivity and specificity of manual and automated measurement of reticulocyte parameters for classification of anemia in dogs: 174 cases (1993-2013). J Am Vet Med Assoc 2016; 249: 776-786.

14. Bauer N, Nakagawa J, Dunker C et al. Evaluation of the automated hematology analyzer Sysmex XT-2000iV ${ }^{\mathrm{TM}}$ compared to the ADVIA ${ }^{\circledR} 2120$ for its use in dogs, 
cats, and horses. Part II: Accuracy of leukocyte differential and reticulocyte count, impact of anticoagulant and sample aging. J Vet Diagn Invest 2012; 24: 74-89.

15. Lilliehöök I and Tvedten H. Validation of the Sysmex XT-2000iV hematology system for dogs, cats, and horses. I. Erythrocytes, platelets, and total leukocyte counts. Vet Clin Pathol 2009; 38: 163-174.|.

16. Gardner IA and Greiner M. Receiver-operating characteristic curves and likelihood Schalm's Veterinary Hematology. $6^{\text {th }}$ ed. Ames, IA: ratios: improvements over traditional methods for the evaluation and application of veterinary clinical pathology tests. Vet Clin Pathol 2006; 35: 8-17.

17. Christenson RH. Evidence-based laboratory medicine - a guide for critical evaluation of in vitro laboratory testing. Ann Clin Biochem 2007; 44: 111-130.

18. Albert A. On the use and computation of likelihood ratios in clinical chemistry. Clin Chem 1982; 28: 1113-1119.

19. Stokol T. Immune-mediated anemias in cats.. In: Weiss DJ and Wardrop KJ (eds). Schalm's Veterinary Hematology. $6^{\text {th }}$ ed. Ames, IA: Wiley-Blackwell, 2010, pp. 226232. cats: features, aetiologies and survival data. J Feline Med Surg. 2013; 15: 81-90. 


\section{Figure legends}

280 Figure 1. Flow diagram illustrating the selection procedure employed in this study.

Figure 2. Distribution of Ret\%, Ret\# and RPI in cats with regenerative anemia (RA) and

284 The boxes indicate the I-III interquartile range (IQR), the horizontal line the median value, and the whiskers extend to further observation within quartile 1 minus $1.5 \mathrm{x} \mathrm{IQR}$ or to further observation within quartile 3 plus $1.5 \mathrm{x}$ IQR. The grey area represents the reference interval of the laboratory. For each parameter and method, results of the RA group were significantly higher $(\mathrm{P}<0.001)$ than results of the NRA group.

Figure 3. Comparison of ROC curves of the Ret\% (gray circle), Ret\# (black circle), and indicates the line of no discrimination. 
293 Table 1: final diagnosis in the 106 cats with regenerative anemia (RA) or non regenerative 294 anemia (NRA) included in this study

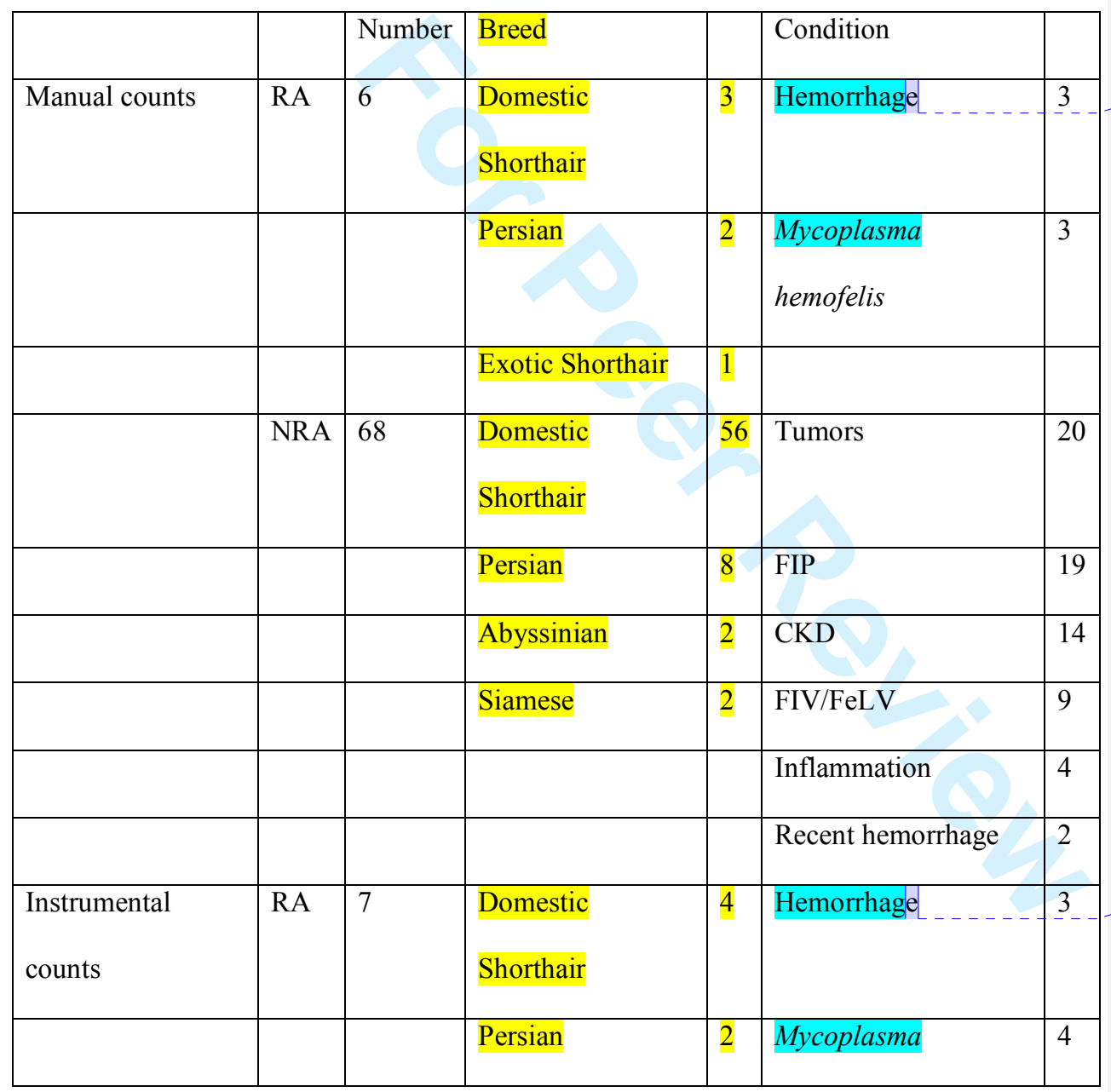

Comment [a67]: Removed: Hemorrhages

Comment [a68]: Rremoved: Hemorrhages 


\begin{tabular}{|l|l|l|l|l|l|l|}
\hline & & & & & hemofelis & \\
\hline & & & Scottish Fold & 1 & & \\
\hline & NRA & 25 & $\begin{array}{l}\text { Domestic } \\
\text { Shorthair }\end{array}$ & 14 & Tumors & 9 \\
\hline & & & Abyssinian & 3 & FIP & 6 \\
\hline & & & Persian & 3 & CKD & 5 \\
\hline & & & Siamese & 2 & FIV/FeLV & 3 \\
\hline & & & Chartreux & 1 & Inflammation & 1 \\
\hline & & & Exovon Rex & 1 & Recent hemorrhage & 1 \\
\hline
\end{tabular}


Table 2. Sensitivity (Sens), specificity (Spec), and positive likelihood ratio (LR+) of Ret\% and Ret\# using pre-determined cut-offs (i.e. the cut-off corresponding to the upper reference limit reported in literature for humans. ${ }^{2}$ ) or using the cut-offs determined by the ROC curve

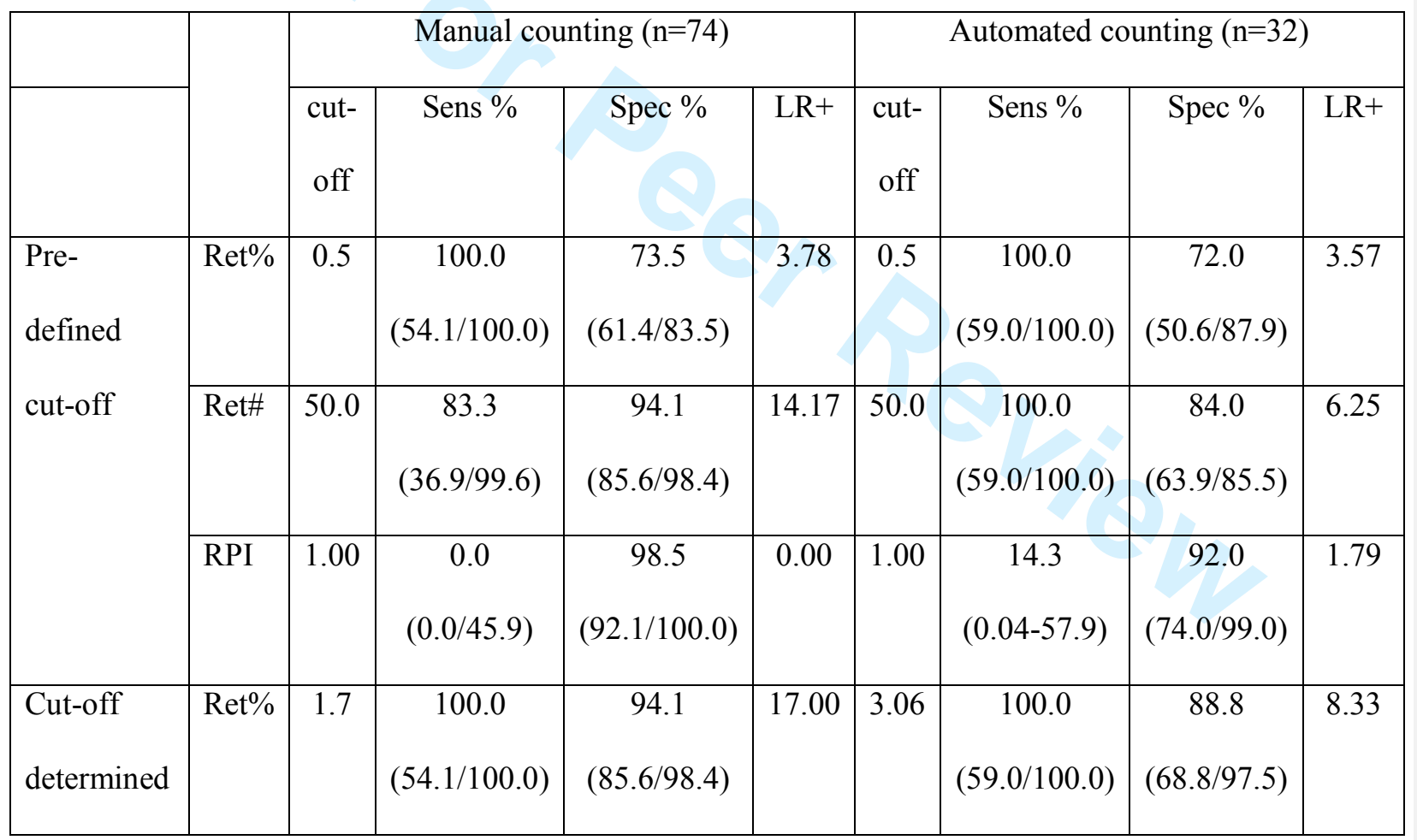




\begin{tabular}{|c|c|c|c|c|c|c|c|c|c|}
\hline \multirow[t]{2}{*}{$\begin{array}{l}\text { by the } \\
\text { ROC curve }\end{array}$} & Ret\# & 41.4 & $\begin{array}{c}100.0 \\
(54.1 / 100.0)\end{array}$ & $\begin{array}{c}92.6 \\
(83.7 / 97.6)\end{array}$ & 13.60 & 57.7 & $\begin{array}{c}100.0 \\
(59.0 / 100.0)\end{array}$ & $\begin{array}{c}88.8 \\
(68.8 / 97.5)\end{array}$ & 8.33 \\
\hline & RPI & 0.35 & $\begin{array}{c}100.0 \\
(54.1 / 100.0)\end{array}$ & $\begin{array}{c}95.6 \\
(87.6 / 99.1)\end{array}$ & 22.67 & 0.59 & $\begin{array}{c}100.0 \\
(59.0 / 100.0)\end{array}$ & $\begin{array}{c}92.0 \\
(74.0 / 99.0)\end{array}$ & 12.50 \\
\hline
\end{tabular}

299

$300 \mathrm{AUC}=$ area under the ROC curve. Values in parentheses are $95 \%$ confidence intervals. 


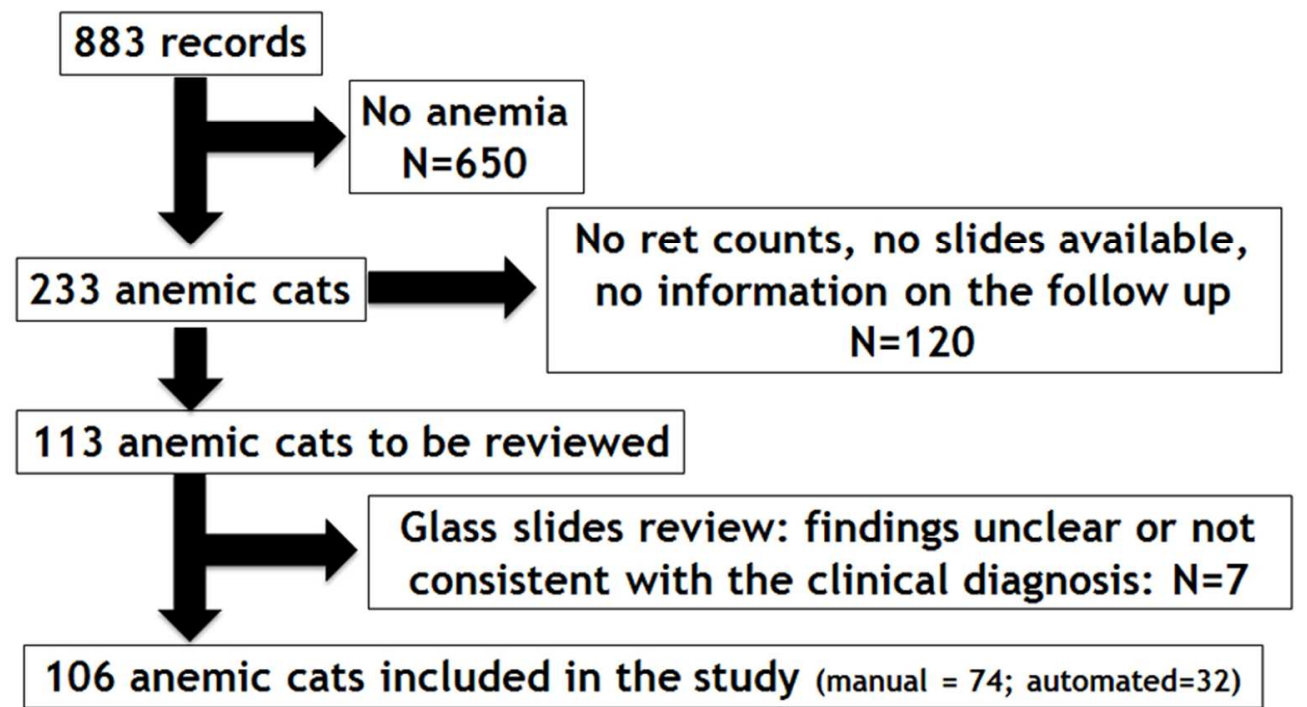

Figure 1. Flow diagram illustrating the selection procedure employed in this study. $80 \times 44 \mathrm{~mm}(300 \times 300$ DPI $)$ 

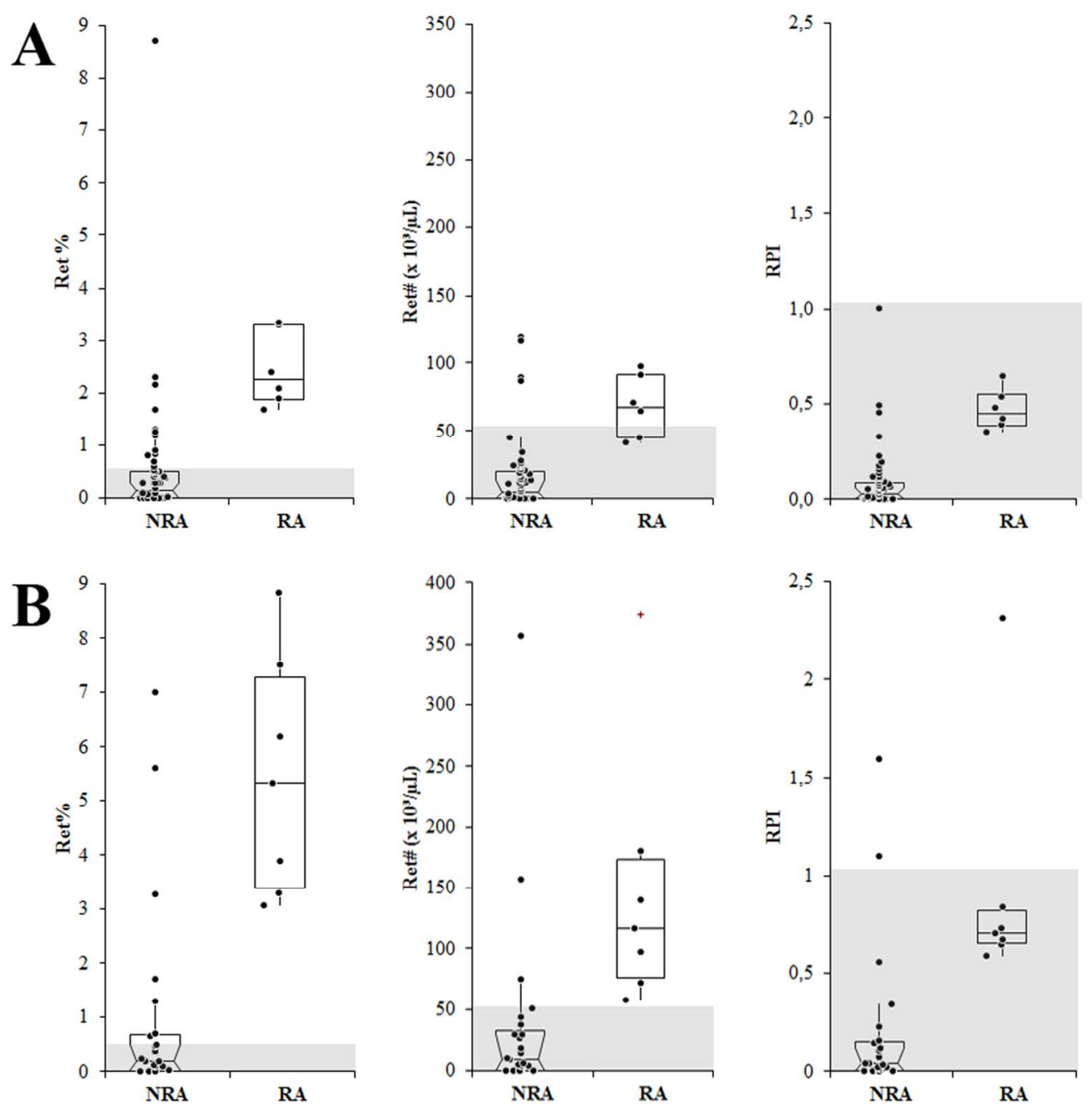

Figure 2. Distribution of Ret\%, Ret\# and RPI in cats with regenerative anemia (RA) and with nonregenerative anemia (NRA), after manual (row A) or automated counts (row B). The boxes indicate the I-III interquartile range (IQR), the horizontal line the median value, and the whiskers extend to further observation within quartile 1 minus $1.5 \times$ IQR or to further observation within quartile 3 plus $1.5 \times$ IQR. The grey area represents the reference interval of the laboratory. For each parameter and method, results of the RA group were significantly higher $(P<0.001)$ than results of the NRA group.

$119 \times 124 \mathrm{~mm}(300 \times 300 \mathrm{DPI})$ 

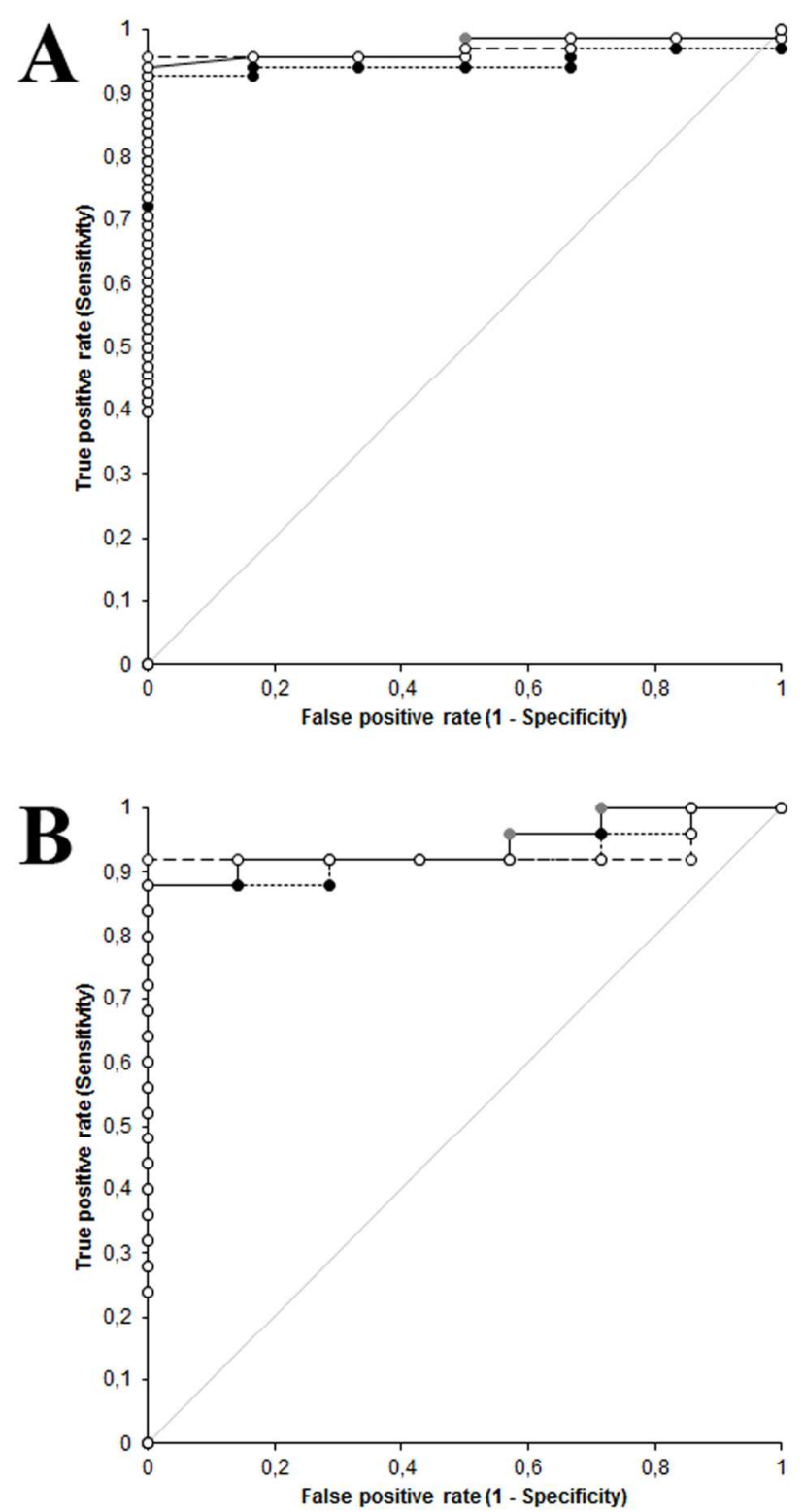

Figure 3. Comparison of ROC curves of the Ret\% (gray circle), Ret\# (black circle), and RPI (open circle) obtained after manual count (A) or instrumental count (B). The gray line indicates the line of no discrimination.

$80 \times 145 \mathrm{~mm}(300 \times 300 \mathrm{DPI})$ 